\title{
MODELING OF THE EIGENFIELD OF A PRESTRESSED HYPERELASTIC MEMBRANE ENCAPSULATING A LIQUID
}

\author{
I. P. GAVRILYUK ${ }^{1}$, M. HERMANN ${ }^{2}$, A. TIMOKHA ${ }^{3}$, AND V. TROTSENKO ${ }^{4}$
}

\begin{abstract}
A spectral boundary problem on the eigenfield of an inflated/deflated stretched circular membrane, which is clamped to a circular cylindrical cavity filled with a liquid, is examined. The paper presents an operator formulation of the problem and proposes a new semi-analytical approximate method. The method captures singular behavior of the solution in the pole and at the fastening contour of the membrane.
\end{abstract}

2000 Mathematics Subject Classification: 35R35, 76B07.

Keywords: hyperelastic membrane, liquid sloshing dynamics, singular functional basis.

\section{Introduction}

The variety of real-world applications dealing with hyperelastic materials to encapsulate a liquid in a cavity is enormous. For example, in the aerospace industry, rubber membranes can cover a propellant in tanks of fuel systems to prevent its fragmentation. In biomechanics, soft vascular tissues may be considered as an isotropic thin-walled vessel (e.g., rubber-like tube), if the blood pressure domain is far below the physiological range (Holzapfel et al. [10]). The list may be continued.

If a hyperelastic membrane is clamped to the edge/walls of a rigid tank filled with a liquid (as considered in the present paper) and ensures the liquid inside, its static shape is very sensitive to fluctuations of the hydrostatic pressure and to the change in the liquid volume. Of course, the simplest case suggests an unstressed membrane with a planar profile. For this case, free oscillations of the "liquid-membrane" system were studied by Siekmann \& Chang [15], Dokuchaev [3], Bauer \& Eidel [1] and Trotsenko [16]. Later, Jiang [12] demonstrated extensions of these results to the case of an equibiaxially-stretched, but yet flat membrane. Increasing/decreasing the mean liquid volume (pressure) due to a slow inlet/outlet through a tank hole can stretch the membrane. In order to describe the stretched membrane diaphragms, a singularly-perturbed boundary value problem should be solved. Its numerical solutions are well known in the literature (see, for instance, Jiang \& Haddow [11] and Trotsenko [17]). The aim of the present paper consists of mathematical and numerical modelling of free oscillations of the "liquid-membrane" system relative to these diaphragm stretched

\footnotetext{
${ }^{1}$ Berufsakademie Thüringen, Staatliche Studienakademie, Am Wartenberg 2, 99817, Eisenach, Germany. E-mail: ipg@ba-eisenach.de

${ }^{2}$ Universität Jena, Ernst-Abbe-Platz. 2-4, 07742, Jena, Germany. E-mail: hermann@mathematik.unijena.de

${ }^{3}$ Centre of Excellence "Centre for Ships and Ocean Structures", Norwegian University of Science and Technology, NO-7491 Trondheim, Norway. E-mail: alexander.timokha@ntnu.no

${ }^{4}$ Institute of Mathematics, National Academy of Sciences, 3 Tereschenkivska St., 01601 Kyiv, Ukraine.
} 
states. To the authors' knowledge, the literature contains neither mathematical, nor numerical results for this problem, though, as shown in the famous experimental studies by Zalesov \& Daev [18], it is relevant, because, being inflated/deflated, the stretched membranes considerably change the eigenfield.

The present paper considers a circular membrane clamped to the edge of a rigid upright circular cylindrical tank filled with an incompressible perfect liquid. The membrane is stretched due to the increase/decrease the liquid volume. It forms an axisymmetric cupola encapsulating the liquid. Bearing in mind the analytical approximations of these stretched diaphragms given by Trotsenko [17], the cupola is assumed to be known a priory. The paper derives a linear evolutional problem that describes small relative coupled oscillations of the "liquid-membrane" system relative the hydrostatic shape. The evolutional problem is re-formulated to an operator differential equation completed with initial conditions, which imply initial perturbations and velocities of the preliminary stretched membrane. Considering the time-harmonic solutions, the Cauchy problem is reduced to a spectral boundary problem on linear natural modes (eigenfunctions). Variational formulation of this spectral problem facilitates the Ritz scheme, whose uniform convergence to the eigenfield needs a special functional basis, which captures asymptotic properties of the eigenfunctions. A series of numerical examples is presented.

\section{Statement of the problem}

2.1. Statically deformed membrane. Let us consider a thin homogeneous hyperelastic membrane of radius $R_{0}$ and thickness $h_{0}\left(h_{0} \ll R_{0}\right)$. The membrane is clamped to the end-side of a rigid circular cylindrical tank. The tank is completely filled with a perfect incompressible liquid. No gas bubbles, concrements, and contamination are assumed in the liquid bulk.

The unstressed (flat) natural reference configuration of the membrane is shown in Fig. 2.1,a. Furthermore, we introduce the geometric set of midpoints (equidistant of the inner

$a$
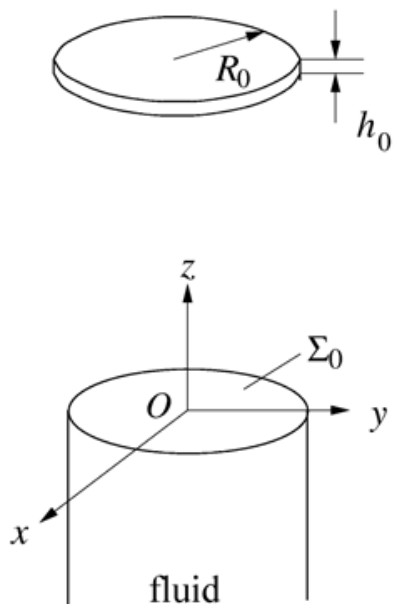

$$
b
$$
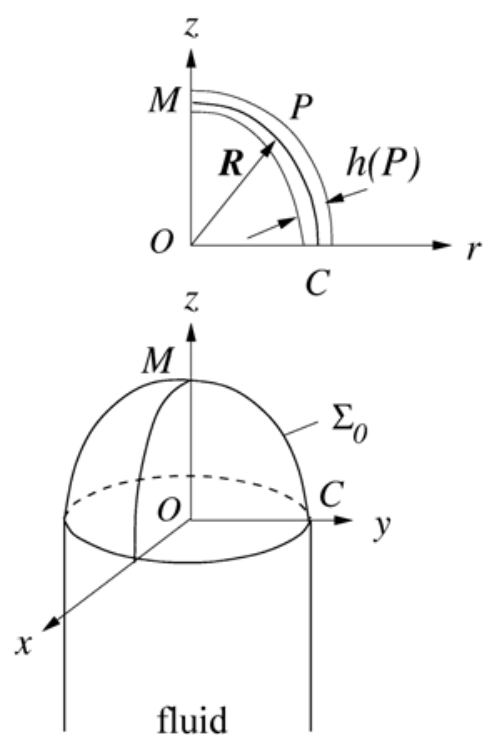

fluid c

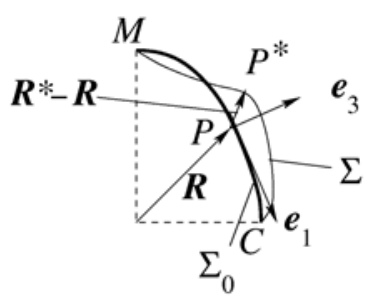

Fig. 2.1. Sketch of the circular membrane and adopted nomenclature. Figure $(a)$ depicts the flat, unperturbed state of the membrane. Figure $(b)$ illustrates an inflated membrane, the cupola. Figure $(c)$ gives some necessary notations in the meridional plane facilitating the problem on small relative oscillations 
and outer sides of the membrane), the surface $\Sigma_{0}$, and superpose it with the $O x y$-plane. The gravitation along the $O z$-axis, or the increase/decrease in the liquid volume stretches the membrane. The inflated/deflated membrane forms the $O z$-symmetric diaphragm exemplified in Fig. 2.1,b. In the last case, the thickness becomes non-constant, but a function of $P \in \Sigma_{0}$, i.e., $h=h(P)$, where $\Sigma_{0}$ is yet the midpoints of the deformed membrane. Describing $\Sigma_{0}$ in the cylindrical coordinate system $O z \eta r$ makes it possible to consider the meridional line $M C$ yielded by the intersection of $\Sigma_{0}$ and the meridional section. Furthermore, the curve $M C$ is parametrised as

$$
\left\{\begin{array}{l}
r=r(s) \\
z=z(s)
\end{array}\right.
$$

where $s$ is the length of $(M P)$. Each point $P \in \Sigma_{0}$ is a function of $s$ and $\eta$, i.e., $P=P(s, \eta)$; the thickness depends on $s$, i.e., $h=h(s)$. If $s_{0}$ defines the end-point $C$ and the pole $M$ corresponds to $s=0$, the following boundary conditions should be fulfilled:

$$
r\left(s_{0}\right)=R_{0} ; \quad z\left(s_{0}\right)=r(0)=z^{\prime}(0)=0 .
$$

The principal stretches, $\lambda_{1}$ and $\lambda_{2}$, tangential to the meridian and the circle of the latitude of $\Sigma_{0}$, given by

$$
\lambda_{1}=\sqrt{\left(\frac{d r(s)}{d s}\right)^{2}+\left(\frac{d z(s)}{d s}\right)^{2}}, \quad \lambda_{2}=\frac{r(s)}{s} \quad \text { and } \quad \lambda_{3}=\frac{1}{\lambda_{1} \lambda_{2}}=\frac{h(s)}{h_{0}}
$$

are the scaled thickness. Further, it is assumed that the inflation/deflation of $\Sigma_{0}$ is imposed to the hydrostatic pressure

$$
Q(z)=C-D z
$$

where the $D$-term implies the gravitation and $C$ is associated with the injection/ejection of the liquid mass through the tank bottom.

In accordance with the geometric elasticity theory (see, Green \& Adkins [9]), the principal components of the Biot stress tensor, $T_{1}$ and $T_{2}$, are defined by

$$
\begin{gathered}
T_{i}=2 h_{0} \lambda_{3}\left(\lambda_{i}^{2}-\lambda_{3}^{2}\right)\left(\frac{\partial W}{\partial I_{1}}+\lambda_{3-i}^{2} \frac{\partial W}{\partial I_{2}}\right), \quad i=1,2, \\
I_{1}=\lambda_{1}^{2}+\lambda_{2}^{2}+\lambda_{3}^{2}, \quad I_{2}=\lambda_{1}^{-2}+\lambda_{2}^{-2}+\lambda_{3}^{-2}
\end{gathered}
$$

where, to express the constitutive relationships for an isotropic hyperelastic solid, the existence of a strain energy function, $W$, should be postulated. In this study, we adopt the four-term strain energy function

$$
W\left(I_{1}, I_{2}\right)=C_{1}\left(I_{1}-3\right)+C_{2}\left(I_{2}-3\right)+C_{3}\left(I_{1}-3\right)^{2}+C_{4}\left(I_{1}-3\right)^{3}
$$

first proposed by Biderman [2] for modeling rubber-like materials. Here, $I_{1}$ and $I_{2}$ are the first and second deviatoric strain invariants defined in (2.5) and $C_{i}(i=1,2,3,4)$ are the experimental constants. In particular cases, where $C_{2}=C_{3}=C_{4}=0,(2.6)$ reduces to the Trealoar law and when $C_{3}=C_{4}=0$, to the two-terms Mooney-Rivlin constitutive law, respectively. 
Based on the Biderman strain energy function (2.6), Trotsenko [17] derived the system of ordinary differential equations coupling $z(s)$ and $r(s)$. Schematically, these equations can be expressed in terms of the principal components, $T_{1}$ and $T_{2}$ from (2.5) as follows:

$$
\frac{d T_{1}}{d s}+\frac{1}{r} \frac{d r}{d s}\left(T_{1}-T_{2}\right)=0, \quad k_{1} T_{1}+k_{2} T_{2}=Q .
$$

Here, $k_{1}$ and $k_{2}$ are the principal curvatures of $\Sigma_{0}$ defined as

$$
k_{1}=\left(\frac{d^{2} r}{d s^{2}} \frac{d z}{d s}-\frac{d r}{d s} \frac{d^{2} z}{d s^{2}}\right) \lambda_{1}^{-3}, \quad k_{2}=-\left(r \lambda_{1}\right)^{-1} \frac{d z}{d s}
$$

System (2.7) contains two fourth-order nonlinear ordinary differential equations with respect to $z(s)$ and $r(s)$. The boundary value problem for system (2.7) should be completed by the boundary conditions (2.2) and the boundedness condition

$$
|z(s)|+|r(s)|<\infty \quad \text { as } \quad s \rightarrow 0 .
$$

2.2. Small relative oscillations. Let us assume that a stretched membrane oscillates with a small amplitude relative to the statically stretched $\Sigma_{0}$ and, thereby, generates internal waves inside of the encapsulated liquid. The instantaneous midpoints of the oscillating membrane $\Sigma(t)$ can be given as

$$
\Sigma(t): \quad \boldsymbol{R}^{*}=\boldsymbol{R}+u \boldsymbol{e}_{1}+v \boldsymbol{e}_{2}+w \boldsymbol{e}_{3}
$$

(see Fig. 2.1,c, where the radius-vector $\boldsymbol{R}$ determines the static equilibrium $\Sigma_{0}, \boldsymbol{e}_{1}$ and $\boldsymbol{e}_{2}$ are tangential unit vectors to the lines of the principal curvature of $\Sigma_{0}, \boldsymbol{e}_{3}$ is the normal unit vector). The functions $u(t, s, \eta), v(t, s, \eta)$ and $w(t, s, \eta)$ are the unknowns implying smallamplitude relative oscillations. Being relatively small, these functions yield linear variations of the Biot stress tensors $\delta T_{1}, \delta T_{2}$ (along the meridians and the parallels) and perturbations of the shear stress $\delta S$.

If $\alpha_{1}=$ const and $\alpha_{2}=$ const define the lines of the principal curvatures, the perturbed equilibrium equation for $\Sigma_{0}$ takes the following form:

$$
\frac{\partial}{\partial \alpha_{1}}\left[B^{*}\left(T_{1}^{*} \boldsymbol{e}_{1}^{*}+\delta S \boldsymbol{e}_{2}^{*}\right)\right]+\frac{\partial}{\partial \alpha_{2}}\left[A^{*}\left(\delta S \boldsymbol{e}_{1}^{*}+T_{2}^{*} \boldsymbol{e}_{2}^{*}\right)\right]+\boldsymbol{Q}^{*} A^{*} B^{*}=0
$$

where $A^{*}, B^{*}$ are the Lame constants and $\boldsymbol{e}_{1}^{*}, \boldsymbol{e}_{2}^{*}, \boldsymbol{e}_{3}^{*}$ are the perturbed unit vectors $\boldsymbol{e}_{1}, \boldsymbol{e}_{2}$ and $\boldsymbol{e}_{3}$, respectively. Analogously, $T_{1}^{*}=T_{1}+\delta T_{1}, T_{2}^{*}=T_{2}+\delta T_{2}$.

The dynamic forces acting on the membrane due to the perturbations relative to $\Sigma_{0}$ take the following form:

$$
\begin{gathered}
\boldsymbol{Q}^{*}=\delta Q_{1} \boldsymbol{e}_{1}^{*}+\delta Q_{2} \boldsymbol{e}_{2}^{*}+\left(Q+\delta Q_{3}^{(1)}+\delta Q_{3}^{(2)}+\delta Q_{3}^{(3)}\right) \boldsymbol{e}_{3}^{*} \\
\delta Q_{1}=-\rho_{0} h \frac{\partial^{2} u}{\partial t^{2}}, \quad \delta Q_{2}=-\rho_{0} h \frac{\partial^{2} v}{\partial t^{2}}, \quad \delta Q_{3}^{(1)}=-\rho_{0} h \frac{\partial^{2} w}{\partial t^{2}}
\end{gathered}
$$

where $\delta Q_{1}, \delta Q_{2}, \delta Q_{3}^{(1)}$ are associated with the inertial features of the membrane ( $\rho_{0}$ is its density). Besides, $\delta Q_{3}^{(2)}$ and $\delta Q_{3}^{(3)}$ express the linear perturbations of the hydrodynamic and hydrostatic components of the pressure, respectively.

Inserting the shear stress and the Lame constants into (2.9) (expressed in terms of (2.1), see Novozhilov [14]) and taking into account the derivatives of $\boldsymbol{e}_{1}, \boldsymbol{e}_{2}, \boldsymbol{e}_{3}$ and the initial 
conditions (2.7), the standard procedure of linearization relative to $\Sigma_{0}$ gives the following scalar equations:

$$
\begin{gathered}
-\frac{1}{\lambda_{1}} \frac{\partial \delta T_{1}}{\partial s}-\left(\delta T_{1}-\delta T_{2}\right) \frac{\cos \alpha}{r}-\frac{1}{r} \frac{\partial \delta S}{\partial \eta}-\frac{T_{10}}{\lambda_{1}} \frac{\partial \varepsilon_{2}}{\partial s}-T_{20}\left[\theta_{1} k_{2}-\frac{1}{r} \frac{\partial \gamma_{2}}{\partial \eta}+\frac{\cos \alpha}{r}\left(\varepsilon_{2}-\varepsilon_{1}\right)\right]=\delta Q_{1}, \\
-\frac{1}{r} \frac{\partial \delta T_{2}}{\partial \eta}-\frac{1}{\lambda_{1}} \frac{\partial \delta S}{\partial s}-2 \delta S \frac{\cos \alpha}{r}-T_{10}\left(\frac{1}{\lambda_{1}} \frac{\partial \gamma_{1}}{\partial s}+\theta_{2} k_{1}\right)-T_{20}\left(\frac{\cos \alpha}{r} \gamma+\frac{1}{r} \frac{\partial \varepsilon_{1}}{\partial \eta}\right)=\delta Q_{2}, \\
k_{1} \delta T_{1}+k_{2} \delta T_{2}+T_{10}\left(\frac{1}{\lambda_{1}} \frac{\partial \theta_{1}}{\partial s}-k_{1} \varepsilon_{1}\right)+T_{20}\left(\frac{\cos \alpha}{r} \theta_{1}+\frac{1}{r} \frac{\partial \theta_{2}}{\partial \eta}-k_{2} \varepsilon_{2}\right)- \\
D(u \sin \alpha-w \cos \alpha)=\delta Q_{3}^{(1)}+\delta Q_{3}^{(2)},
\end{gathered}
$$

where $\alpha$ denotes the angle between the outward normal unit vector to $\Sigma_{0}$ and its symmetry axis and

$$
\begin{gathered}
\varepsilon_{1}=\frac{1}{\lambda_{1}} \frac{\partial u}{\partial s}+k_{1} w, \quad \varepsilon_{2}=\frac{1}{r} \frac{\partial v}{\partial \eta}+\frac{\cos \alpha}{r} u+k_{2} w, \quad \gamma=\gamma_{1}+\gamma_{2} ; \quad \gamma_{1}=\frac{1}{\lambda_{1}} \frac{\partial v}{\partial s}, \\
\gamma_{2}=\frac{1}{r} \frac{\partial u}{\partial \eta}-\frac{\cos \alpha}{r} v, \quad \theta_{1}=-\frac{1}{\lambda_{1}} \frac{\partial w}{\partial s}+k_{1} u, \quad \theta_{2}=-\frac{1}{r} \frac{\partial w}{\partial \eta}+k_{2} v .
\end{gathered}
$$

The dynamic equations (2.10) and (2.11) include the linearized components of the Biot stress tensor and the shear stress

$$
\delta T_{1}=c_{11} \varepsilon_{1}+c_{12} \varepsilon_{2}, \quad \delta T_{2}=c_{21} \varepsilon_{1}+c_{22} \varepsilon_{2}, \quad \delta S=c_{33} \gamma,
$$

where

$$
\begin{gathered}
c_{11}=f_{1}\left(\lambda_{1}, \lambda_{2}\right), \quad c_{12}=f_{2}\left(\lambda_{1}, \lambda_{2}\right), \quad c_{21}=f_{2}\left(\lambda_{2}, \lambda_{1}\right), \quad c_{22}=f_{1}\left(\lambda_{2}, \lambda_{1}\right), \\
c_{33}=2 h_{0}\left[\lambda_{3}^{3} \frac{\partial W}{\partial I_{1}}+\left(\lambda_{1}^{2} \lambda_{3}^{3}+\lambda_{2}^{2} \lambda_{3}^{3}-\lambda_{1} \lambda_{2}\right) \frac{\partial W}{\partial I_{2}}\right], \\
f_{1}\left(\lambda_{1}, \lambda_{2}\right)=2 h_{0}\left[\left(\lambda_{1}^{2} \lambda_{3}+3 \lambda_{3}^{3}\right) \frac{\partial W}{\partial I_{1}}+\left(\lambda_{1} \lambda_{2}+3 \lambda_{2}^{2} \lambda_{3}^{3}\right) \frac{\partial W}{\partial I_{2}}+2 \lambda_{3}\left(\lambda_{1}^{2}-\lambda_{3}^{2}\right)^{2}\left(A_{11}+2 A_{12} \lambda_{2}^{2}+A_{22} \lambda_{2}^{4}\right)\right], \\
f_{2}\left(\lambda_{1}, \lambda_{2}\right)=2 h_{0}\left[\left(3 \lambda_{3}^{3}-\lambda_{1}^{2} \lambda_{3}\right) \frac{\partial W}{\partial I_{1}}+\left(\lambda_{1} \lambda_{2}+\lambda_{2}^{2} \lambda_{3}^{3}\right) \frac{\partial W}{\partial I_{2}}+\right. \\
\left.2 \lambda_{3}\left(\lambda_{1}^{2}-\lambda_{3}^{2}\right)\left(\lambda_{2}^{2}-\lambda_{3}^{2}\right)\left(A_{11}+A_{12}\left(\lambda_{1}^{2}+\lambda_{2}^{2}\right)+A_{22} \lambda_{1}^{2} \lambda_{2}^{2}\right)\right], \quad A_{i k}=\frac{\partial^{2} W}{\partial I_{i} \partial I_{k}}, \quad i, k=1,2 .
\end{gathered}
$$

Using the Bernoulli integral and some straightforward geometric relationships leads to

$$
\delta Q_{3}^{(2)}=-\rho \frac{\partial^{2} \varphi}{\partial t^{2}}, \quad \delta Q_{3}^{(3)}=D(u \sin \alpha-w \cos \alpha),
$$

where $\rho$ is the liquid density and the "displacement" potential $\varphi$ is the solution of the Neumann boundary value problem

$$
\Delta \varphi=0 \quad(z, \eta, r) \in \Omega,\left.\quad \frac{\partial \varphi}{\partial n}\right|_{\Sigma_{0}}=w,\left.\quad \frac{\partial \varphi}{\partial n}\right|_{S}=0, \quad \int_{\Sigma_{0}} w d \Sigma=0
$$


( $S$ is the wetted surface of the tank, $\Omega$ is the liquid volume, $n$ is the outer normal to $\Sigma_{0} \cup S$ ).

System (2.10) includes the unknowns of both geometric and hydrodynamic nature. When using (2.12) and the expressions for (2.11), equations (2.10) can be re-formulated in terms of the displacements $u, v$ and $w$. This generates the following system of linear differential equations:

$$
\begin{gathered}
L_{11}(u)+L_{12}(v)+L_{13}(w)=\delta Q_{1}, \\
L_{21}(u)+L_{22}(v)+L_{23}(w)=\delta Q_{2}, \\
L_{31}(u)+L_{32}(v)+L_{33}(w)=\left(\delta Q_{3}^{(1)}+\delta Q_{3}^{(2)}\right),
\end{gathered}
$$

where

$$
\begin{gathered}
L_{11}(u)=\frac{1}{r \lambda_{1}}\left[-\frac{\partial}{\partial s}\left(\alpha_{1} \frac{\partial u}{\partial s}\right)+\alpha_{2} u-\alpha_{3} \frac{\partial^{2} u}{\partial \eta^{2}}\right], \quad L_{12}(v)=\frac{1}{r \lambda_{1}}\left[\alpha_{4} \frac{\partial^{2} v}{\partial s \partial \eta}+\alpha_{5} \frac{\partial v}{\partial \eta}\right], \\
L_{13}(w)=\frac{1}{r \lambda_{1}}\left[\alpha_{6} \frac{\partial w}{\partial s}+\alpha_{7} w\right], \quad L_{21}(u)=\frac{1}{r \lambda_{1}}\left[\beta_{4} \frac{\partial^{2} u}{\partial s \partial \eta}+\beta_{5} \frac{\partial u}{\partial \eta}\right] \\
L_{22}(v)=\frac{1}{r \lambda_{1}}\left[-\frac{\partial}{\partial s}\left(\beta_{1} \frac{\partial v}{\partial s}\right)+\beta_{2} v-\beta_{3} \frac{\partial^{2} v}{\partial \eta^{2}}\right], \quad L_{23}(w)=\frac{\beta_{6}}{r \lambda_{1}} \frac{\partial w}{\partial \eta}, \\
L_{31}(u)=\frac{1}{r \lambda_{1}}\left[\gamma_{5} \frac{\partial u}{\partial s}+\gamma_{6} u\right], \quad L_{32}(v)=\frac{\gamma_{4}}{r \lambda_{1}} \frac{\partial v}{\partial \eta}, \quad L_{33}(w)=\frac{1}{r \lambda_{1}}\left[-\frac{\partial}{\partial s}\left(\gamma_{1} \frac{\partial w}{\partial s}\right)+\gamma_{2} w-\gamma_{3} \frac{\partial^{2} w}{\partial \eta^{2}}\right], \\
\alpha_{1}=\frac{r c_{11}}{\lambda_{1}}, \quad \alpha_{2}=\alpha_{2}^{(1)}+\frac{d \alpha_{2}^{(2)}}{d s}, \quad \alpha_{2}^{(1)}=\frac{c_{22}}{r \lambda_{1}}\left(\frac{d r}{d s}\right)^{2}-r \lambda_{1} k_{1} k_{2} T_{20}, \quad \alpha_{2}^{(2)}=-\left(\frac{c_{12}+T_{10}}{\lambda_{1}}\right) \frac{d r}{d s}, \\
\alpha_{5}=\alpha_{5}^{(1)}+\frac{d \alpha_{5}^{(2)}}{d s}, \quad \alpha_{5}^{(1)}=\left(c_{33}+c_{22}+T_{20}\right) \frac{1}{r} \frac{d r}{d s}, \quad \alpha_{5}^{(2)}=-\left(c_{12}+T_{10}\right), \quad \alpha_{6}=-r\left(c_{11} k_{1}+c_{21} k_{2}\right), \\
\alpha_{7}=\alpha_{7}^{(1)}+\frac{d \alpha_{7}^{(2)}}{d s}, \quad \alpha_{7}^{(1)}=\left[\left(c_{21}+T_{20}\right) k_{1}+c_{22} k_{2}\right] \frac{d r}{d s}, \quad \alpha_{7}^{(2)}=-r\left[c_{11} k_{1}+\left(c_{12}+T_{10}\right) k_{2}\right], \\
\beta_{1}=\frac{r}{\lambda_{1}}\left(c_{33}+T_{10}\right), \quad \beta_{2}=\beta_{2}^{(1)}+\frac{d \beta_{2}^{(2)}}{d s}, \quad \beta_{2}^{(2)}=\frac{c_{33}}{\lambda_{1}} \frac{d r}{d s}, \quad \beta_{2}^{(1)}=\frac{c_{33}+T_{20}}{r \lambda_{1}}\left(\frac{d r}{d s}\right)^{2}-r \lambda_{1} k_{1} k_{2} T_{10}, \\
\beta_{5}=\beta_{5}^{(1)}+\frac{d \beta_{5}^{(2)}}{d s}, \quad \beta_{5}^{(1)}=-\left(c_{33}+c_{22}+T_{20}\right) \frac{1}{r} \frac{d r}{d s}, \quad \beta_{5}^{(2)}=-c_{33}, \quad \beta_{6}=-\lambda_{1}\left(c_{12} k_{1}-c_{22} k_{2}\right), \\
\gamma_{1}=\frac{r}{\lambda_{1}} T_{10}, \quad \gamma_{2}=r \lambda_{1}\left[\left(c_{11}-T_{10}\right) k_{1}^{2}+\left(c_{22}-T_{20}\right) k_{2}^{2}+\left(c_{12}+c_{21}\right) k_{1} k_{2}\right]+D r \frac{d r}{d s}, \quad \gamma_{3}=\frac{\lambda_{1}}{r} T_{20}, \\
\gamma_{4}=-\beta_{6}, \quad \gamma_{5}=r\left(c_{11} k_{1}+c_{21} k_{2}\right), \\
\gamma_{6}^{(1)}=\left[c_{12} k_{1}+\left(c_{22}-T_{20}\right) k_{2}\right] \frac{d r}{d s}+D r \frac{d z}{d s}, \quad \gamma_{6}^{(2)}=r k_{1} T_{10} .
\end{gathered}
$$

In system (2.15), the functions $u, v$ and $w$ should be bounded at the pole of $\Sigma_{0}$ and, in addition, these must satisfy the clamping conditions on $\partial \Sigma_{0}=l$ :

$$
\left.u\right|_{l}=\left.v\right|_{l}=\left.w\right|_{l}=0 .
$$

Finally, adding the initial conditions for $u, v$ and $w$ leads to an initial-boundary value problem for $(2.14)-(2.16)$. 


\section{Operator formulation}

The evolutional problem $(2.14)-(2.16)$ is scaled by the radius $R_{0}$ so that

$$
\begin{gathered}
\{u, v, w\}=\{\bar{u}, \bar{v}, \bar{w}\} R_{0}, \quad T_{i 0}=\bar{T}_{i 0} \cdot 2 C_{1} h_{0}, \quad i=1,2 \\
a=\frac{\rho R_{0}}{\rho_{0} h_{0}}, \quad \varphi=\bar{\varphi} R^{2}, \quad t=\bar{t} \sqrt{\delta}, \quad \delta=\frac{R_{0}^{2} \rho_{0}}{2 C_{1}}
\end{gathered}
$$

where the bar over the symbols denotes the dimensionless characteristics (furthermore, the bar will be omitted).

Let us consider the Steklov operator $B$

$$
\varphi=B f
$$

which maps $f$ defined on $\Sigma_{0}$ to $\varphi$ found from the Neumann problem (2.14) with $w=f$. In some cases the operator $B$ can be expressed explicitly in terms of the Green function representation, but one should prefer numerical methods to compute its approximation (see, e.g., $[6,7]$ and references therein).

We assume that for any $t$ the vector-function $\boldsymbol{u}=\{u, v, w\}$ belongs to an admissible subset of functions $\mathcal{H}$ from $L_{0}\left(\Sigma_{0}\right)$ that satisfy (2.16) and the volume conservation condition, and equip this set with the scalar product

$$
\left(\vec{u}_{1}, \vec{u}_{2}\right)=\int_{\Sigma_{0}}\left(u_{1} u_{2}+v_{1} v_{2}+w_{1} w_{2}\right) d S
$$

Taking into account the above notations, one can transform the boundary problem $(2.14)-(2.16)$ to the operator differential equation

$$
L \boldsymbol{u}+M \frac{\partial^{2} \boldsymbol{u}}{\partial t^{2}}=0
$$

where $M=\operatorname{diag}\left\{\lambda_{3}, \lambda_{3}, \lambda_{3}+a B\right\}$, and $L$ is the matrix-operator

$$
L=\left\|\begin{array}{lll}
L_{11} & L_{12} & L_{13} \\
L_{21} & L_{22} & L_{23} \\
L_{31} & L_{32} & L_{33}
\end{array}\right\|
$$

defined on $\mathcal{H}$.

The differential equation (3.3) is completed by the initial conditions

$$
\left.\boldsymbol{u}\right|_{t=0}=\boldsymbol{u}_{0}(s, \eta),\left.\quad \frac{\partial \boldsymbol{u}}{\partial t}\right|_{t=0}=\boldsymbol{u}_{0}^{\prime}(s, \eta),
$$

where $\boldsymbol{u}_{0}(s, \eta)$ and $\boldsymbol{u}_{0}^{\prime}(s, \eta)$ are two known vector-functions.

3.1. Properties of $\boldsymbol{M}$. Let us consider $\boldsymbol{u}_{1}, \boldsymbol{u}_{2} \in \mathcal{H}$ and

$$
\left(M \boldsymbol{u}_{1}, \boldsymbol{u}_{2}\right)=\int_{\Sigma_{0}}\left[\lambda_{3} u_{1} u_{2}+\lambda_{3} v_{1} v_{2}+\lambda_{3} w_{1} w_{2}+a\left(B w_{1}\right) w_{2}\right] d S
$$


$1^{\circ}$. Utilizing the Green formula gives

$$
\int_{\Sigma_{0}}\left(B w_{1}\right) w_{2} d S=\int_{\Sigma_{0}} \varphi_{1} \frac{\partial \varphi_{2}}{\partial n} d S=\int_{Q} \nabla \varphi_{1} \nabla \varphi_{2} d Q
$$

and, because

$$
\left(M \boldsymbol{u}_{1}, \boldsymbol{u}_{2}\right)=\int_{\Sigma_{0}}\left[\lambda_{3}\left(u_{1} u_{2}+v_{1} v_{2}+w_{1} w_{2}\right)\right] d S+a \int_{Q} \nabla \varphi_{1} \nabla \varphi_{2} d Q=\left(M \boldsymbol{u}_{2}, \boldsymbol{u}_{1}\right)
$$

$M$ is symmetric and positive.

$2^{\circ}$. There exists an inverse $M^{-1}$ on a set of admissible functions. Following the technique of Kopachevsky \& Krein [13], one can show that the operator $M$ is compact.

3.2. Properties of $\boldsymbol{L}$. Long and tedious derivations make it possible to establish the following features of $L$.

$1^{\circ}$. The line

$$
\begin{gathered}
\left(L \boldsymbol{u}_{1}, \boldsymbol{u}_{2}\right)=\int_{\Sigma_{0}}\left\{c_{11} \varepsilon_{1}^{(1)} \varepsilon_{1}^{(2)}+c_{22} \varepsilon_{2}^{(1)} \varepsilon_{2}^{(2)}+c_{12}\left(\varepsilon_{1}^{(1)} \varepsilon_{2}^{(2)}+\varepsilon_{1}^{(2)} \varepsilon_{2}^{(1)}\right)+c_{33} \gamma^{(1)} \gamma^{(2)}+\right. \\
\left.T_{10}\left(\varepsilon_{1}^{(1)} \varepsilon_{2}^{(2)}+\varepsilon_{1}^{(2)} \varepsilon_{2}^{(1)}+\gamma_{1}^{(1)} \gamma_{1}^{(2)}+\theta_{1}^{(1)} \theta_{1}^{(2)}\right)+T_{20}\left(\theta_{2}^{(1)} \theta_{2}^{(2)}+\gamma_{2}^{(1)} \gamma_{2}^{(2)}\right)\right\} d S- \\
\int_{\Sigma_{0}}\left\{Q \left[k_{1} u^{(1)} u^{(2)}+k_{2} v^{(1)} v^{(2)}+\left(k_{1}+k_{2}\right) w^{(1)} w^{(2)}-\frac{1}{\lambda_{1}}\left(u^{(2)} \frac{\partial w^{(1)}}{\partial s}+u^{(1)} \frac{\partial w^{(2)}}{\partial s}\right)+\right.\right. \\
\left.\left.\frac{1}{r}\left(w^{(2)} \frac{\partial v^{(1)}}{\partial \eta}+w^{(1)} \frac{\partial v^{(2)}}{\partial \eta}\right)\right]-\frac{D}{\lambda_{1}} \frac{d r}{d s} w^{(1)} w^{(2)}\right\} d S=\left(L \boldsymbol{u}_{2}, \boldsymbol{u}_{1}\right)
\end{gathered}
$$

proves that $L$ is symmetric.

Here, $\varepsilon_{i}^{(1)}, \gamma_{i}^{(1)}, \theta_{i}^{(1)}$ and $\varepsilon_{i}^{(2)}, \gamma_{i}^{(2)}, \theta_{i}^{(2)}(i=1,2)$ are defined by $(2.11)$, where $\{u, v, w\}=$ $\left\{u^{(1)}, v^{(1)}, w^{(1)}\right\}$ and $\{u, v, w\}=\left\{u^{(2)}, v^{(2)}, w^{(2)}\right\}$, respectively. Besides, the derivation of (3.5) uses the fact that perturbations occur relative to a static equilibrium, e.g., $c_{21}=c_{12}+T_{10}-T_{20}$ and utilises the formula for integrations by parts

$$
\int_{\Sigma_{0}} \frac{f}{\lambda_{1}} \frac{\partial g}{\partial s} d S=-\int_{\Sigma_{0}} \frac{g}{r \lambda_{1}} \frac{\partial}{\partial s}(r f) d S, \quad \int_{\Sigma_{0}} \frac{f}{r} \frac{\partial g}{\partial \eta} d S=-\int_{\Sigma_{0}} \frac{g}{r} \frac{\partial f}{\partial \eta} d S,
$$

where $f(s, \eta)$ and $g(s, \eta)$ are $2 \pi$-periodic by the second variable and equal to zero on the contour $l\left(s=s_{0}\right)$.

$2^{\circ}$. Assuming $\boldsymbol{u}^{(1)}=\boldsymbol{u}^{(2)}=\boldsymbol{u}$ in (3.5), we arrive at

$$
\begin{gathered}
(L \boldsymbol{u}, \boldsymbol{u})=\int_{\Sigma_{0}}\left\{c_{11} \varepsilon_{1}^{2}+c_{22} \varepsilon_{2}^{2}+2 c_{12} \varepsilon_{1} \varepsilon_{2}+c_{33} \gamma^{2}+T_{10}\left(\gamma_{1}^{2}+\theta_{1}^{2}+2 \varepsilon_{1} \varepsilon_{2}\right)+T_{20}\left(\theta_{2}^{2}+\omega_{2}^{2}\right)\right\} d S- \\
\int_{\Sigma_{0}}\left\{Q\left[k_{1} u^{2}+k_{2} v^{2}+\left(k_{1}+k_{2}\right) w^{2}-\frac{2 u}{\lambda_{1}} \frac{\partial w}{\partial s}+\frac{2 w}{r} \frac{\partial v}{\partial \eta}\right]-\frac{D}{\lambda_{1}} \frac{d r}{d s} w^{2}\right\} d S=2 W, \quad
\end{gathered}
$$

where $W$ is the potential energy. 
As long as the statically stretched membrane $\Sigma_{0}$ is stable and, therefore, $\Sigma_{0}$ corresponds to the non-negative minimum of $W$, equality (3.6) implies that the operator $L$ is positively defined. Besides, there exists a symmetric and pre-compact $L^{-1}$ on $\mathcal{H}$ (see, e.g., [13] ).

3.3 Abstract Cauchy problem. By introducing $\boldsymbol{v}=L^{1 / 2} \boldsymbol{u}$, the operator equation (3.3) can be rewritten in the form

$$
\frac{d^{2} \boldsymbol{v}}{d t^{2}}+A \boldsymbol{v}=0, \quad A=L^{1 / 2} M^{-1} L^{1 / 2}
$$

Here, the operator $A^{-1}=L^{-1 / 2} M L^{1 / 2}$ is self-adjoint, positively defined and compact and, therefore, the Cauchy problem for (3.7) is uniquely solvable for any admissible initial functions $\boldsymbol{v}_{0}$ and $\boldsymbol{v}_{0}{ }^{\prime}$. Solution methods for differential equations with operator coefficients of the type of (3.7) (in time) can be found in $[5,8,13]$

3.4 Natural modes. Another approach very interesting for applications is the investigation of (3.7) in the frequency zone. The problem on natural modes implies the time-harmonic solutions $\boldsymbol{u}=\exp (i \omega t) \boldsymbol{u}(s, \eta)\left(\boldsymbol{v}=L^{\frac{1}{2}} \boldsymbol{u}\right)$. This leads to the spectral problems

$$
L \boldsymbol{u}-\kappa^{2} M \boldsymbol{u}=0
$$

and

$$
A \boldsymbol{v}-\kappa^{2} \boldsymbol{v}=0, \quad \kappa^{2}=\frac{\omega^{2} R_{0}^{2} \rho_{0}}{2 C_{1}},
$$

which have only real positive eigenvalues with the limiting point at infinity. The eigenfunctions (natural modes) of (3.8) and (3.9) constitute the basis in an appropriate space so that, for instance, the orthogonality condition

$$
\left(L \boldsymbol{u}_{i}, \boldsymbol{u}_{j}\right)=\left(M \boldsymbol{u}_{i}, \boldsymbol{u}_{j}\right)=0, \quad i \neq j
$$

should be satisfied. Physically, this means that the coupled "membrane-fluid" oscillations around a stable stretched shape can be decomposed into the sum of standing natural waves.

One can show that (3.8) follows from the necessary extremum condition of the functional

$$
\mathcal{F}(\boldsymbol{u})=(L \boldsymbol{u}, \boldsymbol{u}) /(M \boldsymbol{u}, \boldsymbol{u}) \geqslant 0 .
$$

\section{Approximate solutions}

Since $\Sigma_{0}$ is axisymmetric, the spectral boundary problem (3.8) can be transformed to a family of integro-differential equations by the substitution

$$
u=u_{n} \cos n \eta, \quad v=v_{n} \sin n \eta, \quad w=w_{n} \cos n \eta, \quad \varphi=\varphi_{n} \cos n \eta,
$$

where $n=0,1,2 \ldots$ is the wave number in the angular direction. For the vector-function $\boldsymbol{u}_{n}=\left(u_{n}(s), v_{n}(s), w_{n}(s)\right)$, we get

$$
L_{n} \boldsymbol{u}_{n}-\kappa^{2} M_{n} \boldsymbol{u}_{n}=0, \quad M_{n}=\operatorname{diag}\left\{\lambda_{3}, \lambda_{3}, \lambda_{3}+a H^{(n)}\right\} .
$$

Here, $L_{n}$ is obtained from $L$ by separating the $\eta$-variable, and the functions $\varphi_{n}=H^{(n)} w_{n}$ are solutions of the two-dimensional boundary value problem

$$
\frac{\partial}{\partial r}\left(r \frac{\partial \varphi_{n}}{\partial r}\right)+\frac{\partial}{\partial z}\left(r \frac{\partial \varphi_{n}}{\partial z}\right)-\frac{n^{2}}{r} \varphi_{n}=0, \quad(z, r) \in G,
$$




$$
\left.\frac{\partial \varphi_{n}}{\partial n}\right|_{\Gamma_{1}}=w_{n},\left.\quad \frac{\partial \varphi_{n}}{\partial n}\right|_{\Gamma_{2}}=0, \quad \int_{0}^{1} r \lambda_{1} w_{n} d s=0,
$$

where $G$ is the meridional cross-section of $Q, \Gamma_{1}$ and $\Gamma_{2}$ are the lines formed in the meridional plane by $\Sigma_{0}$ and $S$, respectively.

Using the variational formulation (3.10) reduces (4.2) to

$$
\kappa^{2}=\int_{0}^{1} L_{n} \boldsymbol{u}_{n} \cdot \boldsymbol{u}_{n} r \lambda_{1} d s / \int_{0}^{1} M_{n} \boldsymbol{u}_{n} \cdot \boldsymbol{u}_{n} r \lambda_{1} d s,
$$

so that $\kappa$ corresponds to the stationary points of $\mathcal{F}$.

4.1. Asymptotic behavior of the eigenmodes as $s \rightarrow 0$. The Ritzt method deals with an appropriate functional basis $\left\{u_{i}^{(n)}\right\},\left\{v_{i}^{(n)}\right\}$ and $\left\{w_{i}^{(n)}\right\}$ assumed to be known a priori. The convergence to the solutions should improve if the analytic properties of the functional basis coincide with those for the original eigenfunctions. These properties are studied in the present section.

Let us assume $a=0$ in (4.2) and transform the problem to a more convenient form by defining new functions

$$
y_{1}=u_{n}, \quad y_{2}=v_{n}, \quad y_{3}=w_{n}, \quad y_{4}=s \frac{d u_{n}}{d s}, \quad y_{5}=s \frac{d v_{n}}{d s}, \quad y_{6}=s \frac{d w_{n}}{d s} .
$$

System (4.2) (three second-order differential equations) can be reduced to six equations of the first order

$$
s \frac{d \boldsymbol{y}}{d s}=F \boldsymbol{y}
$$

Here, $\boldsymbol{y}$ is the vector-function with components $y_{i}(i=1,6)$ and the matrix $F$ reads as

$$
F=\left\|\begin{array}{cccccc}
0 & 0 & 0 & 1 & 0 & 0 \\
0 & 0 & 0 & 0 & 1 & 0 \\
0 & 0 & 0 & 0 & 0 & 1 \\
f_{41} & f_{42} & f_{43} & f_{44} & f_{45} & f_{46} \\
f_{51} & f_{52} & f_{53} & f_{54} & f_{55} & 0 \\
f_{61} & f_{62} & f_{63} & f_{64} & 0 & f_{66}
\end{array}\right\|
$$

where

$$
\begin{gathered}
f_{41}=\frac{s^{2}}{\alpha_{1}}\left(\alpha_{2}+n^{2} \alpha_{3}-\kappa^{2} s\right), \quad f_{42}=\frac{n s^{2}}{\alpha_{1}} \alpha_{5} \quad f_{43}=\frac{s^{2}}{\alpha_{1}} \alpha_{7}, \\
f_{44}=-\frac{s^{2}}{\alpha_{1}} \frac{d}{d s}\left(\frac{\alpha_{1}}{s}\right), \quad f_{45}=\frac{n s}{\alpha_{1}} \alpha_{4}, \quad f_{46}=\frac{s}{\alpha_{1}} \alpha_{6}, \\
f_{51}=-\frac{n s^{2}}{\beta_{1}} \beta_{5}, \quad f_{52}=\frac{s^{2}}{\beta_{1}}\left(\beta_{2}+n^{2} \beta_{3}-\kappa^{2} s\right), \quad f_{53}=-\frac{n s^{2}}{\beta_{1}} \beta_{6}, \\
f_{54}=-\frac{n s}{\beta_{1}} \beta_{4}, \quad f_{55}=-\frac{s^{2}}{\beta_{1}} \frac{d}{d s}\left(\frac{\beta_{1}}{s}\right), \\
f_{61}=\frac{s^{2}}{\gamma_{1}} \gamma_{6}, \quad f_{62}=-\frac{n s^{2}}{\gamma_{1}} \beta_{6}, \quad f_{63}=\frac{s^{2}}{\gamma_{1}}\left(\gamma_{2}+n^{2} \gamma_{3}-\kappa^{2} s\right), \quad f_{64}=\frac{s}{\gamma_{1}} \gamma_{5}, \quad f_{66}=-\frac{s^{2}}{\gamma_{1}} \frac{d}{d s}\left(\frac{\gamma_{1}}{s}\right) .
\end{gathered}
$$


Earlier, Trotsenko [17] showed that $z(s)$ and $r(s)$ could be expanded in the Taylor series at $s=0$. The Taylor expansions of $\lambda_{i}(s), T_{i_{0}}(s)$ and $R_{i}(s)(i=1,2)$ include only even powers. One obtains that the functions $f_{i, j}$ are regular at $s=0$, not equal to zero simultaneously and, therefore, $s=0$ is a regular point for system (4.5). Let us pose the matrix $F$ as

$$
F=F_{0}+F_{1} s+F_{2} s^{2}+\ldots,
$$

where $F_{i}$ have the following form

$$
\begin{aligned}
& F_{0}=\left\|\begin{array}{cccccc}
0 & 0 & 0 & 1 & 0 & 0 \\
0 & 0 & 0 & 0 & 1 & 0 \\
0 & 0 & 0 & 0 & 0 & 1 \\
f_{41}^{(0)} & f_{42}^{(0)} & 0 & 0 & f_{45}^{(0)} & 0 \\
f_{51}^{(0)} & f_{52}^{(0)} & 0 & f_{54}^{(0)} & 0 & 0 \\
0 & 0 & f_{63}^{(0)} & 0 & 0 & 0
\end{array}\right\|, \quad F_{2 i-1}=\left\|\begin{array}{cccccc}
0 & 0 & 0 & 0 & 0 & 0 \\
0 & 0 & 0 & 0 & 0 & 0 \\
0 & 0 & 0 & 0 & 0 & 0 \\
0 & 0 & f_{43}^{(2 i-1)} & 0 & 0 & f_{46}^{(2 i-1)} \\
0 & 0 & f_{53}^{(2 i-1)} & 0 & 0 & 0 \\
f_{61}^{(2 i-1)} & f_{62}^{(2 i-1)} & 0 & f_{64}^{(0)} & 0 & 0
\end{array}\right\|, \\
& F_{2 i}=\left\|\begin{array}{cccccc}
0 & 0 & 0 & 0 & 0 & 0 \\
0 & 0 & 0 & 0 & 0 & 0 \\
0 & 0 & 0 & 0 & 0 & 0 \\
f_{41}^{(2 i)} & f_{42}^{(2 i)} & 0 & f_{44}^{(2 i)} & f_{45}^{(2 i)} & 0 \\
f_{51}^{(2 i)} & f_{52}^{(2 i)} & 0 & f_{54}^{(2 i)} & f_{55}^{(2 i)} & 0 \\
0 & 0 & f_{63}^{(2 i)} & 0 & 0 & f_{66}^{(2 i)}
\end{array}\right\|
\end{aligned}
$$

with

$$
\begin{gathered}
f_{41}^{(0)}=\frac{1}{c_{11}^{(0)}}\left[c_{22}^{(0)}+n^{2}\left(c_{33}^{(0)}+T\right)\right], \quad f_{42}^{(0)}=\frac{n}{c_{11}^{(0)}}\left[c_{33}^{(0)}+c_{22}^{(0)}+T\right], \\
f_{45}^{(0)}=-\frac{n}{c_{11}^{(0)}}\left(c_{33}^{(0)}+c_{12}^{(0)}+T\right), \quad f_{51}^{(0)}=\frac{n}{\left(c_{33}^{(0)}+T\right)}\left(c_{33}^{(0)}+c_{22}^{(0)}+T\right), \\
f_{52}^{(0)}=\frac{1}{\left(c_{33}^{(0)}+T\right)}\left(c_{33}^{(0)}+n^{2} c_{22}^{(0)}+T\right), \quad f_{63}^{(0)}=n^{2}, \quad f_{54}^{(0)}=\frac{n}{\left(c_{33}^{(0)}+T\right)}\left(c_{33}^{(0)}+c_{21}^{(0)}+T\right), \\
f_{43}^{(1)}=0, \quad f_{46}^{(1)}=-\frac{c_{11}^{(0)}+c_{21}^{(0)}}{R c_{11}^{0}}, \quad f_{53}^{(1)}=\frac{n \lambda\left(c_{12}^{(0)}+c_{22}^{(0)}\right)}{R\left(c_{33}^{(0)}+T\right)}, \\
f_{61}^{(0)}=\frac{\lambda}{T R}\left(c_{12}^{(0)}+c_{22}^{(0)}\right), \quad f_{62}^{(1)}=n f_{61}^{(1)}, \quad f_{64}^{(1)}=f_{61}^{(1)} .
\end{gathered}
$$

The Taylor expansions of $T_{i 0}, \lambda_{i}$ and $1 / R_{i}(i=1,2)$ need expressions of the second derivatives. These can be found by using equilibrium equations for the statically stretched membrane and take the form

$$
\begin{gathered}
\frac{d^{2} T_{20}}{d s^{2}}=3 \frac{d^{2} T_{10}}{d s^{2}}, \quad \frac{d^{2}}{d s^{2}}\left(\frac{1}{R_{1}}\right)=3 \frac{d^{2}}{d s^{2}}\left(\frac{1}{R_{2}}\right), \quad \frac{d^{2}}{d s^{2}}\left(\frac{1}{R_{2}}\right)=-\frac{1}{T R} \frac{d^{2} T_{10}}{d s^{2}}, \\
\frac{d^{2} \lambda_{1}}{d s^{2}}=-\frac{\lambda^{3}\left(2 \lambda^{6}-3-\Gamma \lambda^{8}\right)}{4 R^{2}\left(\lambda^{6}+3\right)\left(1+\lambda^{2} \Gamma\right)}, \quad \frac{d^{2} \lambda_{2}}{d s^{2}}=\frac{\lambda^{3}\left(2 \lambda^{6}+3+\left(\lambda^{6}+4\right) \Gamma\right)}{4 R^{2}\left(\lambda^{6}+3\right)\left(1+\Gamma \lambda^{2}\right)}, \\
\frac{d^{2} T_{10}}{d s^{2}}=\frac{\lambda^{2}\left[3+\left(\lambda^{6}+2+3 \lambda^{-6}\right) \lambda^{2} \Gamma+\left(\lambda^{6}+2\right) \Gamma^{2} \lambda^{-2}\right]}{2\left(\lambda^{6}+3\right)\left(1+\Gamma \lambda^{2}\right) R^{2}}
\end{gathered}
$$


(derivations utilize $C_{3}=C_{4}=0$ and $\Gamma=C_{2} / C_{1}$ in (2.6); analogous expressions for higher even derivatives can be obtained recursively).

Let us first assume that $n \neq 0$. Because in this case the point $s=0$ remains regular, the local solutions are as follows:

$$
y_{i}=s^{\mu} \sum_{k=0}^{\infty} g_{i, k} s^{k}, \quad i=1, \ldots, 6,
$$

and, using the Cauchy formula, one derives

$$
f_{i \nu} y_{\nu}=\sum_{k=0}^{\infty} \sum_{j=0}^{\infty} g_{\nu, j} f_{i \nu}^{(k-j)} s^{k+\mu}
$$

Substituting (4.6) and (4.7) into (4.5) and comparing the coefficients at $s^{\mu+k}$ leads to the relations

$$
\left[F_{0}-(\mu+k) E\right] \boldsymbol{g}_{k}=\boldsymbol{d}_{k}, \quad k=0,1,2, \ldots,
$$

where $E$ is the identity matrix and $\boldsymbol{g}_{k}=\left\{g_{i, k}\right\}$. The vector $\boldsymbol{d}_{0}$ has only zero-components, but

$$
d_{k}^{(i)}=-\sum_{\nu=1}^{6} \sum_{j=0}^{k-1} g_{\nu, j} f_{i \nu}^{(k-j)}, \quad k=1,2, \ldots, \quad i=1, \ldots, 6 .
$$

Because $\boldsymbol{g}_{0}$ is calculated from the homogeneous system of algebraic equations, nontrivial solutions of (4.8) exist if and only if

$$
\operatorname{det}\left|F_{0}-\mu E\right|=0
$$

for a number $\mu$. Other vectors $\boldsymbol{g}_{k}$ are determined from a recursion arising after inserting the nontrivial solutions into (4.8). Thus, (4.10) yields the secular equation

$\left[\mu^{4}-\mu^{2}\left(f_{41}^{(0)}+f_{52}^{(0)}+f_{45}^{(0)} f_{54}^{(0)}\right)-\mu\left(f_{42}^{(0)} f_{54}^{(0)}+f_{45}^{(0)} f_{51}^{(0)}\right)+\left(f_{41}^{(0)} f_{52}^{(0)}-f_{42}^{(0)} f_{51}^{(0)}\right)\right]\left(\mu^{2}-f_{63}^{(0)}\right)=0$.

Further, account of $f_{i j}^{(0)}$ and $2\left(c_{33}^{(0)}+T\right)-c_{22}^{(0)}+c_{12}^{(0)}=0$ at $s=0$ reduces $(4.11)$ to the form

$$
\left[\mu^{4}-2\left(n^{2}+1\right) \mu^{2}+\left(n^{2}-1\right)\right]\left(\mu^{2}-n^{2}\right)=0
$$

whose roots are

$$
\mu_{1}=n+1, \quad \mu_{2}=n, \quad \mu_{3}=n-1, \quad ; \mu_{4}=-(n-1), \quad \mu_{5}=-n, \quad \mu_{6}=-(n+1) .
$$

Because $\mu_{i}, i=4,5,6$, imply unbounded solutions, we get three admissible solutions of the secular equation.

4.1.1. The case of $\mu=\mu_{1}$. Substituting $\mu_{1}$ into (4.8) and solving these recurrence algebraic problems gives the first family of solutions of (4.5)

$$
y_{1}^{(1)}=s^{n+1} \sum_{k=0}^{\infty} g_{1,2 k}^{(1)} s^{2 k}, \quad y_{2}^{(1)}=s^{n+1} \sum_{k=0}^{\infty} g_{2,2 k}^{(1)} s^{2 k}, \quad y_{3}^{(1)}=s^{n+1} \sum_{k=0}^{\infty} g_{3,2 k+1}^{(1)} s^{2 k+1}
$$

(further superscript at $y_{i}$ and $g_{i, k}$ indicates the family number). 
4.1.2. The case of $\mu=\mu_{2}$. In contrast to the previous case, $\mu_{2}+1$ is also the root of the secular equation for $y_{1}$ and $y_{2}$. The corresponding homogeneous linear problem allows for a nontrivial solution. Generally, we get solutions

$$
y_{1}^{(2)}=s^{n} \sum_{k=0}^{\infty} g_{1,2 k+1}^{(2)} s^{2 k+1}, \quad y_{2}^{(2)}=s^{n} \sum_{k=0}^{\infty} g_{2,2 k+1}^{(2)} s^{2 k+1}, \quad y_{3}^{(2)}=s^{n} \sum_{k=0}^{\infty} g_{3,2 k}^{(2)} s^{2 k} .
$$

4.1.3. The case of $\mu=\mu_{3}$. Let us find solutions of the homogeneous algebraic system (4.8) for $k=0$. Simple analysis for $n=1$ (antisymmetric oscillations of the membrane) gives

$$
g_{1,0}^{(3)}=-g_{2,0}^{(3)}
$$

When $k=1$, the right-hand side of (4.8) becomes zero, but, because $\mu_{3}+1$ is the root of (4.10), problem (4.8) remains resolvable. When $k=2$, we arrive at an inhomogeneous algebraic system with linearly dependent equations, which has a solution leading to the third particular solution of the differential equations (3.10)

$$
y_{1}^{(3)}=s^{n-1} \sum_{k=0}^{\infty} g_{1,2 k}^{(3)} s^{2 k}, \quad y_{2}^{(3)}=s^{n-1} \sum_{k=0}^{\infty} g_{2,2 k}^{(3)} s^{2 k}, \quad y_{3}^{(3)}=s^{n-1} \sum_{k=0}^{\infty} g_{3,2 k+1}^{(3)} s^{2 k+1} .
$$

Hence, in general, we have got three linearly independent formal solutions for $u_{n}(s), v_{n}(s)$ and $w_{n}(s)$, which have the form

$$
u_{n}(s)=s^{n-1} \varphi_{n, 1}(s), \quad v_{n}(s)=s^{n-1} \varphi_{n, 2}(s), \quad w_{n}(s)=s^{n} \varphi_{n, 3}(s) .
$$

Here $\varphi_{n, 1}, \varphi_{n, 2}$ and $\varphi_{n, 3}$ are smooth functions which can be expanded into a Taylor series containing only even powers. In the case that $n=1$, the first three coefficients in the power expansions for $\varphi_{n, 1}$ and $\varphi_{n, 2}$ have equal but countersigned absolute values.

In a similar way for $n=0$ one shows that the solutions $u_{0}(s)$ and $w_{0}(s)$ are formally presented as

$$
u_{0}=s \sum_{k=0}^{\infty} a_{k} s^{2 k}, \quad w_{0}=\sum_{k=0}^{\infty} b_{k} s^{2 k}
$$

4.2. Approximate solutions. Let us construct approximate analytical solutions of the boundary value problem $(4.2)$ by using the functional $\mathcal{F}$. In order to do that, $u_{n}(s), v_{n}(s)$ and $w_{n}(s)$ are posed as the truncated series

$$
u_{n}(s)=\sum_{k=1}^{p} x_{k} u_{k}^{(n)}(s), \quad v_{n}(s)=\sum_{k=1}^{p} x_{k+p} v_{k}^{(n)}(s), \quad w_{n}(s)=\sum_{k=1}^{p} x_{k+2 p} w_{k}^{(n)}(s),
$$

where $\left\{u_{k}^{(n)}(s)\right\},\left\{v_{k}^{(n)}(s)\right\}$ and $\left\{w_{k}^{(n)}(s)\right\}$ are the corresponding basis functions.

We will construct a polynomial functional basis that satisfies the boundary conditions at $s=s_{0}$ and has asymptotic behavior as $s \rightarrow 0$ (given by (4.14) and (4.15)). In addition, in studying axisymmetric oscillations, the system $\left\{w_{k}^{(0)}\right\}$ should be restricted to the additional equation

$$
\int_{0}^{1} r \lambda_{1} w_{k}^{(0)}(s) d s=0
$$

following from the volume conservation condition. 
For $n \geqslant 1$, the functions $u_{k}^{(n)}(s), v_{k}^{(n)}(s)$ and $w_{k}^{(n)}(s)$ take the following form:

$$
u_{k}^{(n)}(s)=\left(s^{2}-1\right) s^{n+2 k-3}, \quad w_{k}^{(n)}(s)=\left(s^{2}-1\right) s^{n+2 k-2}, \quad v_{k}^{(n)}(s)=u_{k}^{(n)}(s), \quad k=1, \ldots, p .
$$

Besides, in the case that $n=1, x_{1+p}=-x_{1}$ in expansions (4.16) $x_{1+p}=-x_{1}$. In the case that $n>1$, starting from (4.4) gives the $3 p$-component vector $\boldsymbol{x}=\left\{x_{1}, x_{2}, \ldots, x_{3 p}\right\}$ to be found from the homogeneous algebraic system

$$
\begin{gathered}
\left(A_{n}-\kappa^{2} B_{n}\right) \boldsymbol{x}=0 . \\
a_{i, j}^{(n)}=\int_{0}^{1}\left[\alpha_{1} \frac{d u_{j}^{(n)}}{d s} \frac{d u_{i}^{(n)}}{d s}+\left(\alpha_{2}^{(1)}+n^{2} \alpha_{3}\right) u_{i}^{(n)} u_{j}^{(n)}-\alpha_{2}^{(2)} \frac{d}{d s}\left(u_{i}^{(n)} u_{j}^{(n)}\right)\right] d s, \\
a_{i, j+p}^{(n)}=\int_{0}^{1} n\left[\alpha_{4} \frac{d v_{j}^{(n)}}{d s} u_{i}^{(n)}+\alpha_{5}^{(1)} u_{j}^{(n)} u_{i}^{(n)}-\alpha_{5}^{(2)} \frac{d}{d s}\left(v_{j}^{(n)} u_{i}^{(n)}\right)\right] d s, \\
a_{i, j+2 p}^{(n)}=\int_{0}^{1}\left[\left(\alpha_{6} \frac{d w_{j}^{(n)}}{d s}+\alpha_{7}^{(1)} w_{j}^{(n)}\right) u_{i}^{(n)}-\alpha_{7}^{(2)} \frac{d}{d s}\left(w_{j}^{(n)} u_{j}^{(n)}\right)\right] d s, \\
a_{i+p, j+p}^{(n)}=\int_{0}^{1}\left[\beta_{1} \frac{d v_{j}^{(n)}}{d s} \frac{d v_{i}^{(n)}}{d s}+\left(\beta_{2}^{(1)}+n^{2} \beta_{3}\right) v_{i}^{(n)} v_{j}^{(n)}-\beta_{2}^{(2)} \frac{d}{d s}\left(v_{i}^{(n)} v_{j}^{(n)}\right)\right] d s, \\
\int_{0}^{1}\left[\gamma_{1} \frac{d w_{i}^{(n)}}{d s} \frac{d w_{j}^{(n)}}{d s}+\gamma_{2}^{(n)} w_{i}^{(n)} w_{j}^{(n)}+n^{2} \gamma_{3} w_{i}^{(n)} w_{j}^{(n)}\right] d s, \quad i, j=1, \ldots, p . \\
a_{i+2 p, j+2 p}^{(n)}=-n v_{i}^{(n)} w_{j}^{(n)} d s,
\end{gathered}
$$

The matrix $B_{n}$ has a block-diagonal structure with the following non-zero elements:

$b_{i j}^{(n)}=\int_{0}^{1} s u_{i}^{(n)} u_{j}^{(n)} d s, \quad b_{i+p, j+p}^{(n)}=\int_{0}^{1} s v_{i}^{(n)} v_{j}^{(n)} d s, \quad b_{i+2 p, j+2 p}^{(n)}=\int_{0}^{1}\left(s+a r \lambda_{1} H^{(n)}\right) w_{i}^{(n)} w_{j}^{(n)} d s$.

In the case that $n=1$ (asymmetric wave profiles), the dimension of the algebraic system (4.18) decreases by 1 . The matrices are obtained from the matrices $A_{n}$ and $B_{n}$ by crossing-out the $(p+1)$-row and the $(p+1)$-column with simultaneous replacement of the corresponding elements by the values

$$
\begin{gathered}
a_{1,1}^{(1)}=\int_{0}^{1}\left[\left(\alpha_{1}+\beta_{1}\right)\left(\frac{d u_{1}^{(1)}}{d s}\right)^{2}+\left(\alpha_{2}^{(1)}+\beta_{2}^{(1)}+\alpha_{3}+\beta_{3}-2 \alpha_{5}^{(1)}\right)\left(u_{1}^{(1)}\right)^{2}+\right. \\
\left.2 u_{1}^{(1)} \frac{d u_{1}^{(2)}}{d s}\left(2 \alpha_{5}^{(2)}-\alpha_{4}-\alpha_{2}^{(2)}-\beta_{2}^{(2)}\right)\right] d s
\end{gathered}
$$




$$
\begin{gathered}
a_{1, j}^{(1)}=\int_{0}^{1}\left[\alpha_{1} \frac{d u_{1}^{(1)}}{d s} \frac{d u_{j}^{(1)}}{d s}+\left(\alpha_{2}^{(1)}-\alpha_{5}^{(1)}+\alpha_{3}\right)+u_{1}^{(1)} u_{j}^{(1)}-\alpha_{4}^{(1)} \frac{d u_{1}^{(1)}}{d s} u_{j}^{(1)}+\left(\alpha_{5}^{(2)}-\alpha_{2}^{(2)}\right) \frac{d}{d s}\left(u_{1}^{(1)} u_{j}^{(1)}\right)\right] d s, \\
a_{1, j+p-1}^{(1)}=\int_{0}^{1}\left[-\beta_{1} \frac{d u_{1}^{(1)}}{d s} \frac{d u_{j}^{(1)}}{d s}+\left(\alpha_{5}^{(1)}-\beta_{2}^{(1)}-\beta_{3}\right) u_{1}^{(1)} u_{j}^{(1)}+\alpha_{4} u_{1}^{(1)} \frac{d u_{j}^{(1)}}{d s}+\left(\beta_{2}^{(2)}-\alpha_{5}^{(2)}\right) \frac{d}{d s}\left(u_{1}^{(1)} u_{j}^{(1)}\right)\right] d s, \\
a_{1, j+2 p-1}^{(1)}=\int_{0}^{1}\left[\left(\alpha_{6}-\alpha_{7}^{(2)}\right) \frac{d w_{j}^{(1)}}{d s} u_{1}^{(1)}+\left(\alpha_{7}^{(1)}+\beta_{6}\right) w_{j}^{(1)} u_{1}^{(1)}-\alpha_{7}^{(2)} w_{j}^{(1)} \frac{d u_{1}^{(1)}}{d s}\right] d s, \\
b_{1,1}^{(1)}=2 \int_{0}^{1} s\left(u_{1}^{(1)}\right)^{2} d s, \quad b_{1, j+p-1}^{(1)}=-\int_{0}^{1} s u_{1}^{(1)} u_{j}^{(1)} d s, \quad j=2, \ldots, p .
\end{gathered}
$$

In considering axisymmetric oscillations $(n=0)$, the elements of the matrices $A_{0}$ and $B_{0}$ are computed by formulae (4.19), (4.20), and the $p$-central row should be crossed out. It should be noted that applying the formula of integration by parts to $A_{n}$ makes it possible to avoid higher derivatives in the coefficients $\alpha_{2}, \alpha_{5}, \alpha_{7}, \beta_{2}, \beta_{5}, \beta_{6}$. In order to compute $B_{n}$, the function $\varphi_{n}^{(i)}=H^{(n)} w_{i}^{(n)}$ should be known on the contour $\Gamma_{1}$. This suggests solutions of the Neumann boundary problems

$$
\begin{gathered}
\frac{\partial}{\partial r}\left(r \frac{\partial \varphi_{n}^{(i)}}{\partial r}\right)+\frac{\partial}{\partial z}\left(r \frac{\partial \varphi_{n}^{(i)}}{\partial z}\right)-\frac{n^{2}}{r} \varphi_{n}^{(i)}=0, \quad(z, r) \in G, \\
\left.\frac{\partial \varphi_{n}^{(i)}}{\partial n}\right|_{\Gamma_{1}}=w_{i}^{(n)},\left.\quad \frac{\partial \varphi_{n}^{(i)}}{\partial n}\right|_{\Gamma_{2}}=0, \quad i=1, \ldots, p .
\end{gathered}
$$

In contrast to (3.9), the boundary condition on the contour $\Gamma_{1}$ contains the already known functions.

The variational formulation of (4.21) reduces the problem to the minimization of the functional

$$
I=\int_{G}\left\{r\left[\left(\frac{\partial \varphi_{n}^{(i)}}{\partial z}\right)^{2}+\left(\frac{\partial \varphi_{n}^{(i)}}{\partial r}\right)^{2}\right]-\frac{n^{2}}{r}\left(\varphi_{n}^{(i)}\right)^{2}\right\} d z d r-2 \int_{\Gamma_{1}} r w_{i}^{(n)} \varphi_{n}^{(i)} d s .
$$

The variational problem can be solved by the Ritzt method. The solutions $\varphi_{n}^{(i)}(z, r)$ are given as follows:

$$
\varphi_{n}^{(i)}(z, r)=\sum_{k=1}^{m} a_{k}^{(i)} V_{k}^{(n)}(z, r)
$$

where $a_{k}^{(i)}$ are the unknowns, $V_{k}^{(n)}(z, r)$ is the system of linearly independent solutions of (4.21). The necessary extremum condition of (4.22) (by $a_{k}^{(i)}$ ) leads to the system of linear inhomogeneous algebraic equations in $\boldsymbol{a}^{(i)}=\left\{a_{1}^{(i)}, a_{2}^{(i)}, \ldots, a_{m}^{(i)}\right\}$

$$
D \boldsymbol{a}^{(i)}=\gamma^{i}, \quad i=1, \ldots, p .
$$


Using the Green formula establishes the following expressions for the matrix $D=\left\{d_{k l}\right\}$ and vectors $\gamma^{i}$ :

$$
d_{k l}=\int_{\Gamma} r \frac{\partial V_{k}^{(n)}}{\partial n} V_{l}^{(n)} d S, \quad \gamma_{k}^{(i)}=\int_{\Gamma_{1}} r V_{k}^{(n)} w_{i}^{(n)} d S, \quad k, l=1, \ldots, m, \quad \Gamma=\Gamma_{1} \cup \Gamma_{2} .
$$

The functional basis $\left\{V_{k}^{(n)}(z, r)\right\}$ coincides with the linearly independent solutions of equation (4.21) given in the polar coordinate system $R$ and $\theta$ by Feschenko et al. [4].

If the domain is occupied by a liquid, it is confined to the rigid walls and the statically inflated membrane, the coordinate functions $V_{k}^{(n)}(z, r)$ can be chosen as

$$
V_{k}^{(n)}(z, r)=W_{k}^{(n)}(z, r)=\frac{2^{n} n !(k-n) !}{(k+n) !} R^{k} P_{k}^{(n)}(\cos \theta), \quad R=\sqrt{z^{2}+r^{2}}, \quad k \geqslant n
$$

where $P_{k}^{(n)}(\cos \theta)$ are the Legendre functions of the first kind.

When the membrane $\Sigma_{0}$ is deflated, the origin does not belong to $G$ and the set $W_{k}^{(n)}(z, r)$ should be completed by the functions

$$
\bar{W}_{k}^{(n)}(z, r)=\frac{2^{n} n !(k-n) !}{(k+n) !} R^{-(k+1)} P_{k}^{(n)}(\cos \theta)=W_{k}^{(n)}(z, r) / R^{2 k+1},
$$

which have a power singularity in the origin. It should be noted that the computations of $W_{k}^{(n)}(z, r), \bar{W}_{k}^{(n)}(z, r)$ and of their derivatives are facilitated by using the recurrence formulated by Feschenko et al. [4].

Thus, the computation of $\varphi_{n}^{(i)}(z, r), i=1, \ldots, p$, reduces to the calculation of $D$ and the $p$-vectors $\gamma^{(i)}$ by the sequence of linear inhomogeneous algebraic equations (4.24). The elements of the matrices $B_{k}$ in (42) that take into account the influence of the liquid, can be found by a supplementary routine splitting of the solution into partial derivatives for the potential and the system of ordinary differential equations on the interval associated with the membrane.

4.3. Numerical examples. Let us consider a vertical circular cylindrical tank of radius $R_{0}$ filled with a liquid to the depth $H / R_{0}=0.5$ so that the free surface is covered by a membrane. The dimensional geometrical and physical characteristics are chosen as follows:

$$
\begin{gathered}
R_{0}=1 \mathrm{~m}, \quad h_{0}=2 \cdot 10^{-3} \mathrm{~m}, \quad C_{1}=93.195 \cdot 10^{-4} \mathrm{~N} / \mathrm{m}^{2}, \quad C_{2}=17.168 \cdot 10^{-4} \mathrm{~N} / \mathrm{m}^{2}, \\
C_{3}=0, \quad C_{4}=0 .
\end{gathered}
$$

The corresponding dimensionless values are $D=2,631579, \Gamma=C_{2} / C_{1}=0.184211$. The parameter $C$ depends on the variation of the liquid volume $\Delta V$ as follows

$$
\Delta V=\pi \int_{0}^{1} r^{2} \frac{d z}{d s} d s
$$

Furthermore, we will change $C$ and restore $\Delta V$. Suppose that the liquid volume increases. Define the value of $C$ to be equal to $0.2 ; 0.6 ; 1.4$. This makes $\Delta V / \pi$ equal to 0.055641 ; $0.124403 ; 0.219157$. 
If the parameter $C$ is relatively small, the major part of the static membrane $\Sigma_{0}$ is close to its homogeneous strained state. The surface is nearly flat except for the small domain localized at the fastening contour. In this case, the nonlinear static problem on $\Sigma_{0}$ belongs to the so-called singularly perturbed problems (Trotsenko [17]). Increasing $C$ increases the strains and makes this area of the membrane larger (see, Fig. 4.1). Let us present some results on free oscillations of a statically deformed membrane coupled with the liquid.

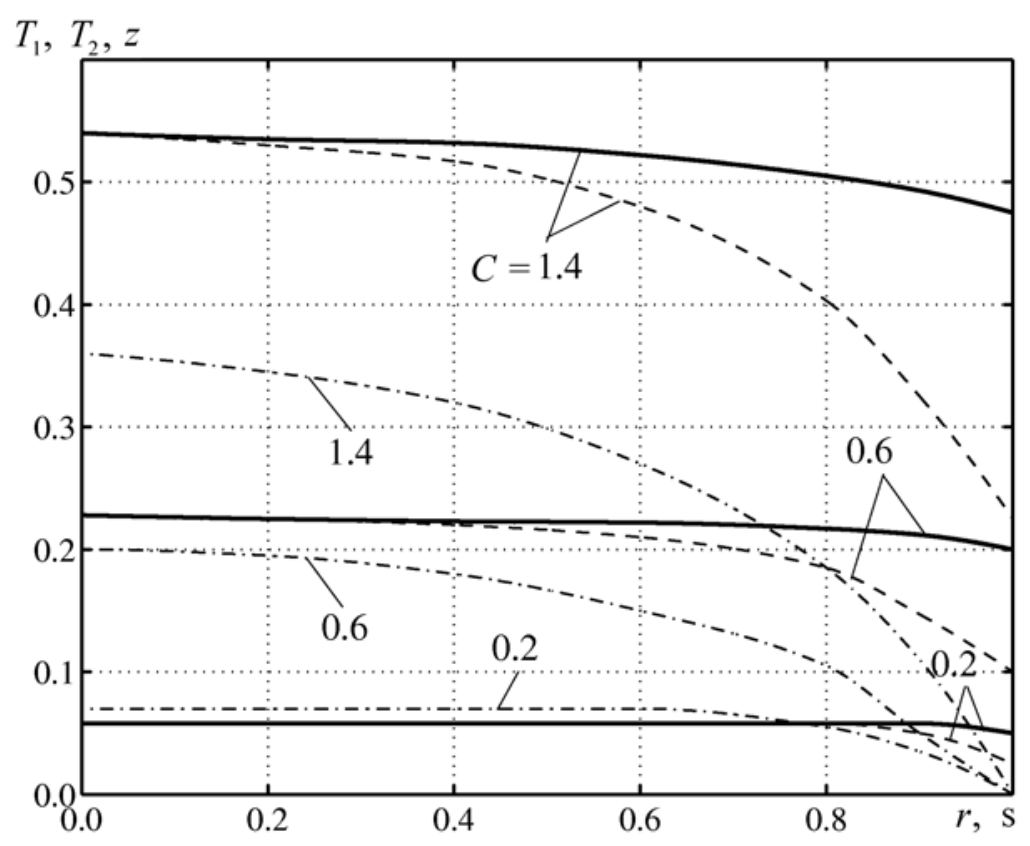

Fig. 4.1. Profiles (dashed lines) and strains of the deformed membrane for the given parameters of hydrostatic loads

Table 4.1 shows the convergence of the first three spectral parameters $\kappa_{i}^{2}$ for the case that $n=1$ versus the number of approximations $p$ in (4.16) with a fixed number of functions in (4.23) $(m=10)$. Here, we supposed $C=1.4 ; \rho / \rho_{0}=1 ; R_{0} / h_{0}=500$ and inertia of the membrane in the tangential direction was neglected. The influence of $m$ in (4.23) (for the potential components) on the accuracy of $\kappa_{i}^{2}$ is shown in Table 4.2. The number of terms in (4.16) for the deviation components was assumed to be five. The results on the change in the eigenfrequencies $\omega$ (normalized) versus $\Delta V$ are presented in Fig. 4.2.

Thus, the numerical data obtained demonstrate the efficiency of the proposed approximate method. The fast convergence to the solution is facilitated by a functional basis of a specific singular structure.

Table 4.1.

Table 4.2 .

\begin{tabular}{|c|c|c|c|}
\hline$p$ & $\kappa_{1}^{2} \cdot 10$ & $\kappa_{2}^{2}$ & $\kappa_{3}^{2}$ \\
\hline 1 & 0.38495 & - & - \\
2 & 0.36532 & 0.27951 & - \\
3 & 0.35860 & 0.23283 & 1.09715 \\
4 & 0.35844 & 0.23097 & 0.74836 \\
5 & 0.35843 & 0.23096 & 0.72226 \\
6 & 0.35843 & 0.23096 & 0.72189 \\
\hline
\end{tabular}

\begin{tabular}{|c|c|c|c|}
\hline$m$ & $\kappa_{1}^{2} \cdot 10$ & $\kappa_{2}^{2}$ & $\kappa_{3}^{2}$ \\
\hline 4 & 0.36024 & 0.42981 & - \\
6 & 0.35851 & 0.23944 & - \\
8 & 0.35847 & 0.23097 & 0.81385 \\
10 & 0.35843 & 0.23096 & 0.72226 \\
12 & 0.35843 & 0.23096 & 0.72207 \\
\hline
\end{tabular}

Acknowledgement. Part of the work was supported the German Research Council (DFG). A. Timokha acknowledges the financial support provided by the Alexander von Humboldt Foundation. 


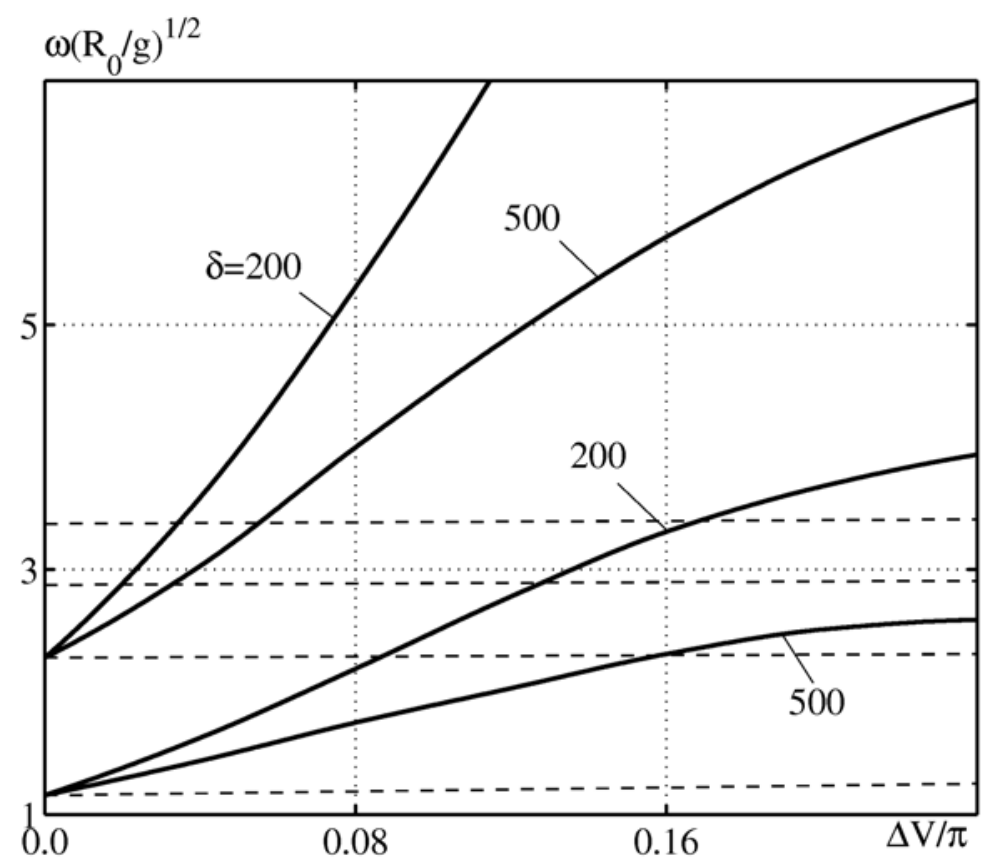

F ig. 4.2. Graphic dependence of the first two values of dimensionless $\omega \sqrt{R / g}$ versus $\Delta V / \pi$ with $\delta=R_{0} / h_{0}$ equal to 200 and 500 . The dashed line corresponds to the first four dimensionless natural sloshing frequencies of the liquid in a circular cylindrical tank. When $\Delta V \rightarrow 0$, the eigenfrequencies of the "liquidmembrane" system tend to the natural sloshing frequencies. As would be expected, growth of $\Delta V$ leads not only to deformations of the membrane, but also to larger natural frequencies of coupled oscillations

\section{References}

1. H. F. Bauer and W. Eidel, Non-linear hydroelastics in rectangular containers, J. Sound and Vibrations, 125 (1988), no. 1, pp. 93-114.

2. V.A.Biderman, Questions on computing rubber-like details, Calculations of Safety, (1958), no. 3, pp. 40-87.

3. L. V. Dokuchaev, On oscillations of a container with a fluid the free surface of which is covered by a membrane, Building Mechanics and Computing the Structures, 1 (1972), pp. 49-54. (in Russian)

4. S. F. Feschenko, I. A. Lukovsky, B. I. Rabinovich, and L. V. Dokuchaev, Methods for computing added masses of a liquid in mobile cavities, Naukova dumka, Kiev, 1969. (in Russian)

5. I.P. Gavrilyuk, Strongly P-positive operators and explicit representations of the solutions of initial value problems for second order differential equations in Banach space, Journal of Math. Anal. and Appl., 236 (1999), pp. 327-349.

6. I. P. Gavrilyuk, W. Hackbusch, and B. Khoromskij, H-matrix approximation for elliptic solution operators in cylindric domains, East-West Journal of Numerical Analysis, 9 (2001), no. 1, pp. 25-58.

7. I. P. Gavrilyuk, A.B. Kulyk, and V.L. Makarov, Integral Equations of the Linear Sloshing in an Infinite Chute and Their Discretization, Computational Methods in Applied Mathematics, 1 (2001), no.1, pp. 39-61.

8. I.P. Gavrilyuk and V.L. Makarov, Explicit and approximate solutions of second order evolution differential equations in Hilbert space, Numerical Methods for Partial Differential Equations, 15 (1999), pp. 111-131.

9. A. E. Green and J. E. Adkins, Large Elastic Deformations, 2nd edn, Clarendon Press, Oxford, 1970.

10. G. A. Holzapfel, R. Eberlein, P. Wriggers, and H. W. Weizsäcker, Large strain analysis of soft biological membranes: Formulation and finite element analysis, Comput. Methods Appl. Engrg., 132 (1996), pp. $45-61$. 
11. L. Jiang and J. B. Haddow, A finite element formulation for finite static axisymmetric deformation of hyperelastic membranes, Computers \& Structures, 57 (1995), no. 3, pp. 401-405.

12. L. Jiang, Application of the Taylor-Galerkin finite element and Godunov-type finite difference methods to finite amplitude axisymmetric waves in hyperelastic membranes, J. Sound and Vibration, 192 (1996), no. 1, pp. 223-244.

13. N. D. Kopachevsky, S. G. Krein, Operator approach to linear problems of hydrodynamics. Vol. 1. Self-adjoint problems for an ideal fluid, Operator Theory: Advances and Applications, Vol. 128. Birkhäuser Verlag, Basel, 2001.

14. V. V. Novozhilov, Theory of thin shells, Sudprogiz, Leningrad, 1958. (in Russian)

15. J.Siekmann and S. C. Chang, On the change of natural frequencies of a sloshing liquid by movable devices, Acta Mechanica, 11 (1971), no. 1-2, pp. 73-86.

16. V.A. Trotsenko, Free oscillations in a rectangular channel with an elastic membrane on the free surface, International Applied Mechanics, 31 (1995), no. 8, pp. 74-80.

17. V. A. Trotsenko, Variational methods for solving nonlinear boundary problems of statics of hyperelastic membranes, Journal of Nonlinear Mathematical Physics, 6 (1999), no. 1, pp. 35-50.

18. V. K. Zalesov and I. F. Daev, Plastic deformation of ejecting diaphragms, Mashinostroenie, Moscow, 1977. (in Russian) 